\title{
Spectroscopy of BL Lacertae objects of extraordinary luminosity
}

\author{
M. Landoni ${ }^{1,2}$, R. Falomo ${ }^{3}$, A. Treves ${ }^{4,2}$, and B. Sbarufatti ${ }^{1}$ \\ 1 INAF - Osservatorio Astronomico di Brera, via Bianchi 46, 23807 Merate (LC), Italy \\ e-mail: marco.landoni@uninsubria.it \\ 2 INAF - Istituto Nazionale di Astrofisica and INFN - Istituto Nazionale di Fisica Nucleare, Italy \\ 3 INAF - Osservatorio Astronomico di Padova, Vicolo dell' Osservatorio 5, 35122 Padova, Italy \\ ${ }^{4}$ Università degli Studi dell'Insubria, via Valleggio 11, 22100 Como, Italy \\ Received 19 May 2014 / Accepted 10 July 2014
}

\section{ABSTRACT}

\begin{abstract}
Aims. We aim to determine the redshift (or stringent lower limits) of a number of bright BL Lacs objects.
Methods. We secured optical and near-infrared medium-resolution spectra of four bright BL Lac objects of unknown redshift using the spectrograph X-Shooter at the ESO-VLT.

Results. In spite of the high quality of the spectra and the extended spectral range of the observations, we have not detected intrisic spectral features for these sources. However, we are able to provide strigent lower limits to their redshift. In particular, for the two TeV sources PG $1553+113$ and $\mathrm{H} 1722+119$, we infer $z>0.30$ and $z>0.35$ respectively. We also detect an intervening Ca II absorption doublet in the spectrum of MH 2136-428 that is ascribed to the halo of a nearby giant elliptical galaxy at a projected distance of $\sim 100 \mathrm{kpc}$.

Conclusions. Under the hypothesis that all BL Lacs are hosted by a luminous bulge-dominated galaxies, the spectroscopic observations of bright BL Lacs presently indicate that these objects are likely sources with extremely beamed nuclear emission. We present simulations to show under which circumstances it is possible to probe this hypothesis from the detection of very weak absorptions using the next generation of extremely large optical telescopes.
\end{abstract}

Key words. BL Lacertae objects: general - instrumentation: spectrographs - cosmology: observations

\section{Introduction}

BL Lac objects are active nuclei of massive elliptical galaxies characterized by a strong non-thermal emission that is dominated by a relativistic jet, which is closely aligned along the line of sight (see e.g. Falomo et al., in prep.). As a consequence, their optical spectra exhibit weak lines or a featureless continuum thus preventing the determination of their redshift. Nevertheless, the estimated distance of BL Lac objects is mandatory for constraining models of their emission, considering that these objects are also the dominant population of the extragalactic sky at high energies, especially for the $\mathrm{TeV}$ band (e.g. Costamante 2013). In the absence of direct redshift measurement through the nuclear emission lines or absorptions from host galaxies, other procedures have been proposed. Some are based on the measurement of the absolute magnitude of the host galaxy, which can be assumed as a standard candle (see Sbarufatti et al. 2005a, and references therein), while others exploit the detection of intervening absorption lines toward the source to set a lower limit to the redshift. For instance, this method was successfully exploited with HST/COS observations of Lyman- $\alpha$ forest in far-ultraviolet (FUV) constraining the redshift of a number of bright BL Lacs at $z \sim 0.4$ (see e.g. Danforth et al. 2010; Furniss et al. 2013). Another approach to obtain redshift lower limits of BLL is to use their spectral energy distribution over a very large spectral range (Rau et al. 2012).

* Figures 6-17 are available in electronic form at http://www . aanda.org

$\star \star$ The full spectra are only available at the CDS via anonymous ftp to cdsarc.u-strasbg.fr $(130.79 .128 .5)$ or via

http://cdsarc.u-strasbg.fr/viz-bin/qcat?J/A+A/570/A126
The improved signal-to-noise ratio $(\mathrm{S} / \mathrm{N})$ of optical spectra collected through a new generation large telescopes, combined with high throughput instruments, has also been successfully adopted in the determination of redshifts of BL Lacs (e.g. Sbarufatti et al. 2006; Landoni et al. 2013; Shaw et al. 2013, and references therein). The campaigns carried out by Sbarufatti et al. (2006) and Landoni et al. (2013) at the 10mt class telescopes, VLT with FORS2 spectrograph, which improves the S/N of up to $\sim 300$ for bright sources allowed the determination of redshifts for many objects that had featureless spectra based on observations collected by $4 \mathrm{mt}$ class telescopes.

In this context, the new generation of spectrographs that combine high resolution and wide spectral coverage yield a considerable improvement on these issues. In this light, the state-ofthe-art X-Shooter spectrograph at ESO-VLT is the most natural choice to tackle BL Lac spectroscopy. With this instrument, it is possible to detect very faint spectral features $(E W \sim 1$ order of magnitude smaller than those detectable with previous instruments). This translates into the possibility to infer a stringent lower limit to the redshift of the sources in which no spectral features (intrinsic or due to intervening systems) are present.

To exploit these new capabilities we obtained spectroscopy of five bright BL Lac objects $(R \leq 17)$, which had defeated previous redshift determinations. They are, therefore, expected to be extremely luminous in the optical band or extremely beamed. In this work we report the results for four sources since PKS 0048097 was already discussed in Landoni et al. (2012).

The paper is organised as follows. Our observations and data reduction are detailed in Sect. 2 while main spectroscopical results are reported in Sect. 3. Discussion and conclusions on the 
Table 1. Journal of observations.

\begin{tabular}{|c|c|c|c|c|c|c|c|}
\hline Object $^{a}$ & Date of observation $^{b}$ & Seeing $^{c}$ & Slit width ${ }^{d}$ & $R^{e}$ & $t_{\exp }^{f}$ & $N^{g}$ & Channel $^{h}$ \\
\hline & & & $1.6^{\prime \prime} \times 11^{\prime \prime}$ & 3300 & 2720 & 4 & (UVB) \\
\hline \multirow{2}{*}{ PG $1553+113$} & 28 Apr 2010 & 1.30 & $1.5^{\prime \prime} \times 11^{\prime \prime}$ & 5400 & 2460 & 6 & (VIS) \\
\hline & & & $1.5^{\prime \prime} \times 11^{\prime \prime}$ & 3500 & 1440 & 6 & (NIR) \\
\hline \multirow{3}{*}{ H $1722+119$} & & & $1.6^{\prime \prime} \times 11^{\prime \prime}$ & 3300 & 2720 & 4 & (UVB) \\
\hline & 28 Apr 2010 & 1.60 & $1.5^{\prime \prime} \times 11^{\prime \prime}$ & 5400 & 2460 & 6 & (VIS) \\
\hline & & & $1.5^{\prime \prime} \times 11^{\prime \prime}$ & 3500 & 1440 & 6 & (NIR) \\
\hline \multirow{3}{*}{ MH 2136-428 } & & & $1.6^{\prime \prime} \times 11^{\prime \prime}$ & 3300 & 2720 & 4 & (UVB) \\
\hline & 28 Apr 2010 & 1.40 & $1.5^{\prime \prime} \times 11^{\prime \prime}$ & 5400 & 2460 & 6 & (VIS) \\
\hline & & & $1.5^{\prime \prime} \times 11^{\prime \prime}$ & 3500 & 1440 & 6 & (NIR) \\
\hline \multirow{3}{*}{ PKS 2254-204 } & & & $1.6^{\prime \prime} \times 11^{\prime \prime}$ & 3300 & 5440 & 4 & (UVB) \\
\hline & 30 Ago / 06 Sep 2010 & 1.20 & $1.5^{\prime \prime} \times 11^{\prime \prime}$ & 5400 & 4920 & 6 & (VIS) \\
\hline & & & $1.5^{\prime \prime} \times 11^{\prime \prime}$ & 3500 & 2880 & 6 & (NIR) \\
\hline
\end{tabular}

Notes. ${ }^{(a)}$ IAU name. ${ }^{(b)}$ Date of observation. ${ }^{(c)}$ Seeing during the observation (measured on the acquisition images). ${ }^{(d)}$ Slit width (in arcsec). ${ }^{(e)}$ Average Resolution $(\lambda / \delta \lambda) .{ }^{(f)}$ Total integration time (in seconds). ${ }^{(g)}$ Number of spectra obtained in nodding mode. ${ }^{(h)}$ X-Shooter Channel.

Table 2. Redshift lower limits and properties of observed sources.

\begin{tabular}{lcccccccc}
\hline \hline Object $^{a}$ & $R^{b}$ & $E W_{\min }{ }^{c}$ & $S / N^{d}$ & $\alpha^{e}$ & $M_{R}{ }^{f}$ & $N / H$ ratio $^{g}$ & $z^{h}$ & $\delta_{\text {opt }}{ }^{i}$ \\
\hline PG 1553+113 & 13.80 & 0.04 & $140 \pm 30$ & 0.90 & $\leq-27.2$ & 50 & $\geq 0.30$ & $\geq 700$ \\
H 1722+119 & 14.90 & 0.07 & $80 \pm 20$ & 1.00 & $\leq-26.3$ & 25 & $\geq 0.35$ & $\geq 400$ \\
MH 2136-428 & 15.80 & 0.06 & $100 \pm 30$ & 0.75 & $\leq-26.0$ & 15 & $\geq 0.50$ & $\geq 500$ \\
PKS 2254-204 & 17.10 & 0.10 & $60 \pm 10$ & 0.75 & $\leq-25.4$ & 10 & $\geq 0.65$ & $\geq 300$ \\
\hline
\end{tabular}

Notes. ${ }^{(a)}$ IAU name. ${ }^{(b)} R$ band apparent magnitude measured from our spectrum. ${ }^{(c)}$ Average EW min in the range $4000-8000 \AA .{ }^{(d)}$ Average $\mathrm{S} / \mathrm{N}$ in the range 4000-8000 $\AA .{ }^{(e)}$ Spectral index. ${ }^{(f)}$ Nucleus absolute magnitude limit in $R$ band computed assuming lower limit on the $z .{ }^{(g)}$ Lower limit of nucleus-to-host ratio by assuming BL Lac host galaxy standard candle with $M_{r}=-22.90 .{ }^{(h)}$ Redshift lower limit. ${ }^{(i)}$ Optical beaming factor computed by following the procedure described in Landoni et al. (2013).

overall sample with an outlook on future perspective are given in Sect. 4. Throughout the paper, we consider the following cosmological parameters $H_{0}=70 \mathrm{~km} \mathrm{~s}^{-1} \mathrm{Mpc}^{-1}, \Omega_{\mathrm{m}}=0.27$ and $\Omega_{\Lambda}=0.73$.

\section{Observations, data reduction and analysis}

We secured UVB (3200-5500 ̊), VIS (5600-10000 ̊) and NIR (11000-15000 $\AA$ ) spectra of the targets in service mode using the European Southern Observatory (ESO) Very Large Telescope (VLT) equipped with the X-Shooter spectrograph (Vernet et al. 2011). The instrument was configured in standard NODDING mode by adopting the recommended ABBA sequence for the exposures to guarantee an accurate sky subtraction in the extracted frames. The journal of observation is reported in Table 1.

Data reduction was performed by the X-Shooter data reduction pipeline (version 2.0.0, see Goldoni et al. 2006) in polynomial mode. In particular, we calculated the master bias frames and master flat frames for each channel for each observation. We also obtained the bidimensional mapping required by the pipeline stack to resample the echelle orders. Finally, we computed the sensitivity functions to calibrate the spectra in flux using spectrophotometric standard stars for each channel.

To consider slit loss effects and overall systematics on the flux calibration of the spectra, we performed a photometry on the acquisition images of the targets, that were secured in $R$ band. The overall uncertainty on the flux (based on the estimation of the $\mathrm{S} / \mathrm{N}$ inside the aperture considered for photometry) is $\Delta m \sim 0.20$, while the wavelength calibration is accurate within $\Delta \lambda \sim 0.5 \AA$. Checking the weather conditions at Paranal, the targets have been observed under clear sky conditions with the exception of PG 1553+113 and, marginally, H 1722+119 due to thin clouds during the exposure. The acquisition images have been secured with clear sky.

From the calibrated spectra of each source, we first compute the minimum detectable equivalent width (EW) by following the procedure described in Sbarufatti et al. (2005b) and we search for spectral features with an EW greater than the minimum measurable one. In the case of absence of detectable features, we exploit the capability of the instrument to measure minimal EW in the range 4000-8000 $\AA$ in order to put stringent lower limit to sources, which exhibit totally featureless spectra. We evaluate the $E W_{\min }$ on the wavelength range $4000-8000 \AA$, since it corresponds to a redshift interval of about $0.01 \leq z \leq 1.00$ for the most prominent absorption lines of the host galaxy. This choice appears reasonable because is challenging, even with medium resolution spectroscopy with a reasonable $\mathrm{S} / \mathrm{N}$ for $8 \mathrm{mt}$ class telescopes, the detection of the underling host galaxy if the object lies at redshift higher than $z \geq 1$, while even higher redshifts are also ruled out by the absence of Ly- $\alpha$ forest in the UV part of the X-Shooter spectrum. The minimum EW on the spectra, the apparent magnitude of the nucleus and the redshift are related through the equation:

$E W_{\mathrm{obs}}=\frac{(1+z) E W_{0}}{1+\rho_{0} \Delta(\lambda) A(z)}$,

where $E W_{\text {obs }}$ is the observed minimum equivalent width; $E W_{0}$ is the equivalent width of the feature in the host galaxy template of Kinney et al. (1996); $\rho_{0}$ is the nucleus-to-host flux ratio in the rest frame $R$ band effective wavelength and $A(z)$ is the aperture correction. The term $\Delta(\lambda)$ is a normalisation factor, which considers the redshift of the source. The knowledge of $E W_{\text {obs }}$, which is the detectable minimum one, and the apparent magnitude of the nucleus allow us to apply Eq. (1) to derive a lower limit to 
H 1722+119 X-Shooter spectrum
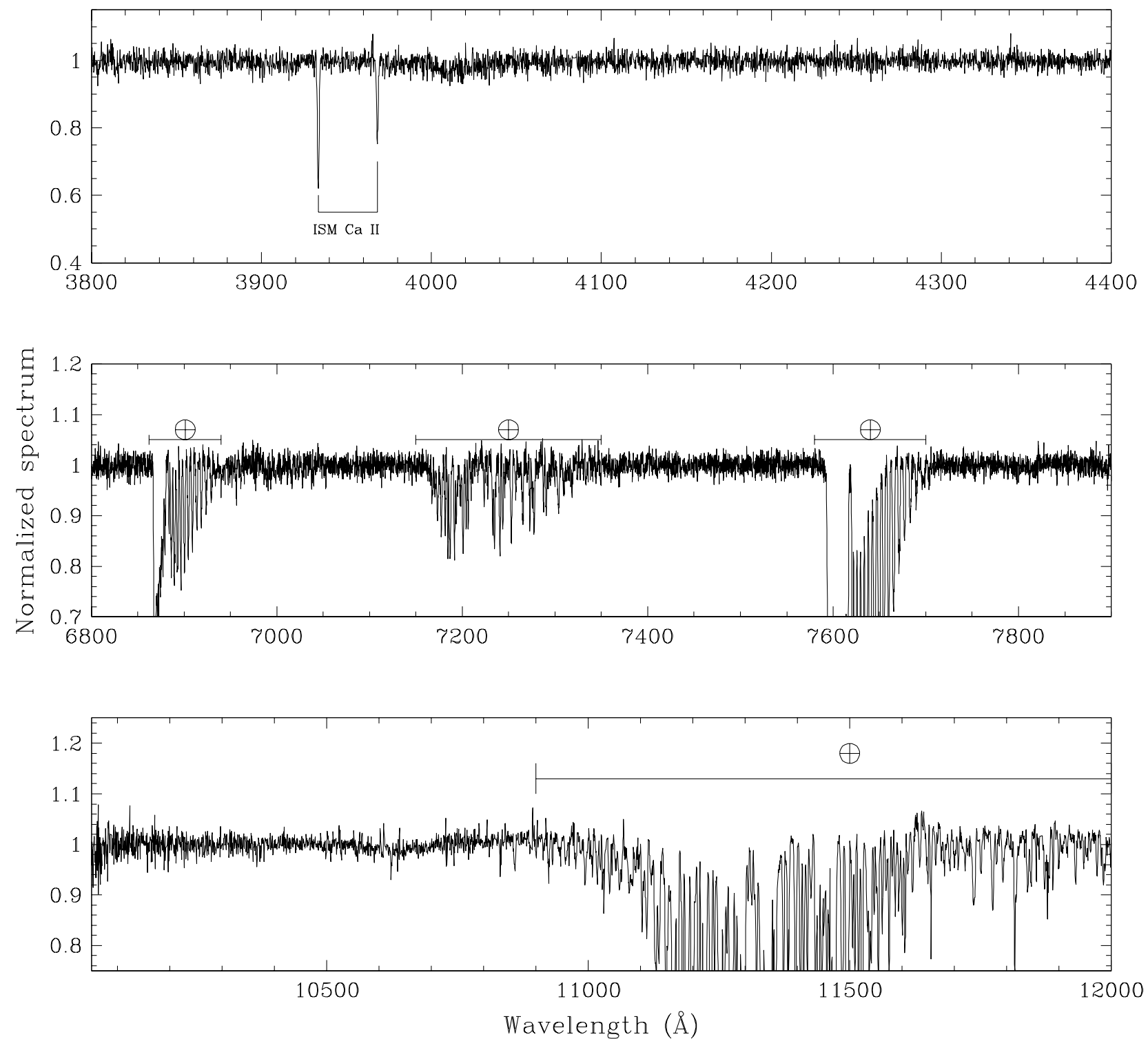

Fig. 1. H 1722+119 X-Shooter normalised spectrum in three sample ranges (first panel: UVB channel; middle panel: VIS channel; bottom panel: near-IR channel). With the exception of galactic and telluric features, no intrinsic or intervening spectral lines are detected in the full range of $\sim 0.33-1.5 \mu \mathrm{m}$.

the redshift. Details are given in Sbarufatti et al. (2006) and references therein. We applied this procedure to our four spectra, which are reported in the results of Table 2.

Finally, to take advantage of the available extended spectral range, we performed a synthetic narrow band photometry by integrating the flux density in various bins of $200 \AA$ on the spectrum of each object aimed to construct the spectral energy distribution (SED).

\section{Results}

We report an example of the spectra in three different ranges from optical to near-IR in Fig. $1^{1}$. With the exception of

\footnotetext{
1 The full spectra for the four sources (Figs. 6-17) are available in both the electronic edition of the paper and on the website database http://archive.oapd.inaf.it/zbllac/
}

MH 2136-428 where we are able to detect an intervening Ca II system at $z \sim 0.008$ (see section notes on individual sources), the other spectra do not show features apart from the galactic and telluric ones. The lower limit to the redshift for each source are also reported in Table 2.

In Fig. 2, we show the SEDs obtained with the procedure as described in Sect. 2. The observed emission is the sum of two components: a non-thermal power, which arises from the relativistic jet (single power law), and host galaxy starlight contribution at $z \sim 0.5$ from a luminous giant elliptical of $M_{r}=-23.90$, which is $\sim 1$ mag more luminous than a typical BL Lac host galaxy (giant elliptical of $M_{r}=-22.90$, Sbarufatti et al. 2005a). As illustrated in Fig. 2, the emission of each source is completely dominated by the non-thermal component and the starlight contribution from the galaxy, despite its luminosity, is negligible. 

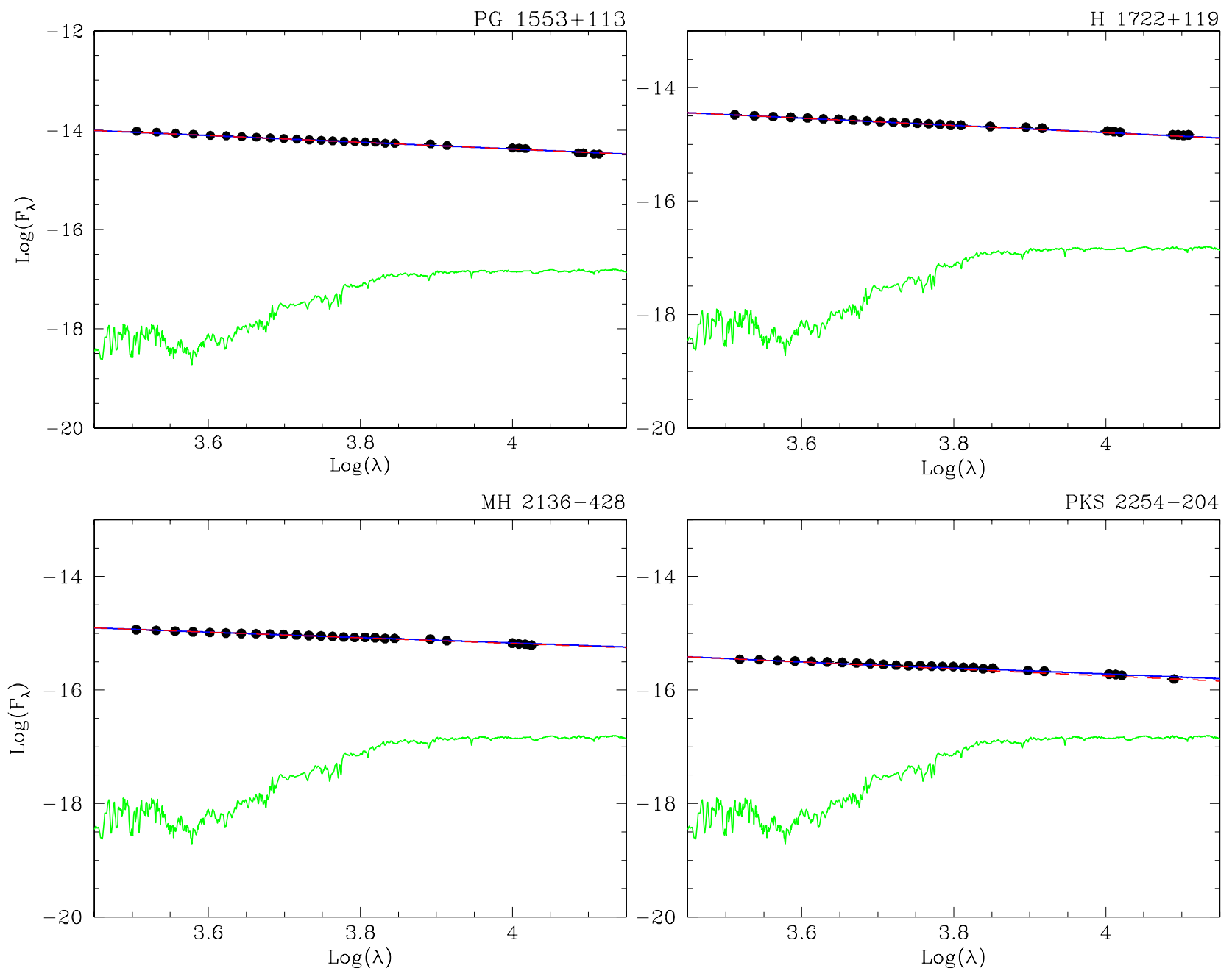

Fig. 2. SEDs of the four bright BL Lac object obtained from X-Shooter spectra in the wavelength range 3200-15000 $\AA$. The blue solid line is the sum of the two components: the non-thermal emission, which is described by a single power law (red dashed line), and the contribution from a luminous host galaxy starlight $\left(M_{r}=-23.90\right)$ at $z=0.50$ (green solid line). The superposition of the blue and red lines indicate that such a galaxy is hidden by the very bright nuclear emission.

\subsection{Notes on individual sources}

PKS 1553+113: this very bright $(R \sim 14)$ BL Lac source, which appeared in the Palomar-Green catalogue of ultraviolet-excess stellar objects (Green et al. 1986), has been thoroughly studied from the radio band to very high energy (VHE, we mention the recent availability of Cherenkov telescopes observations from e.g. Prandini et al. 2010 and Aleksić et al. 2012). In the near UV and optical bands (see e.g. Falomo \& Treves 1990; Falomo et al. 1993), its spectrum appears to be featureless and dominated by the non-thermal emission. Spectroscopy with an $8 \mathrm{~m}$ class telescope at VLT (Sbarufatti et al. 2006) also confirmed a featureless nature of the spectrum and derived a lower limit for the source of $z>0.1$ based on the lack of absorption features from the starlight component. The non-detection of a standard host galaxy in the HST images (Treves et al. 2007) allowed us to estimate $z \geq 0.30-0.40$ making it one of the most distant $\mathrm{TeV}$ extragalactic source. These limits are consistent with two recent constraints on the redshift. The first is obtained through far UV spectroscopy with HST/COS $(z \sim 0.40-0.50$, see Danforth et al. 2010) and exploits the Lyman- $\alpha$ absorption systems toward the line of sight of the source, while the other is based on the observed absorption of the high energy $\mathrm{TeV}$ due to photon-photon interaction with the extragalactic background light, yielding $z \leq 0.60$ (see Prandini et al. 2010). The X-Shooter spectroscopy presented in this paper confirms the featureless nature of the spectrum on the wide spectral range 3200-15000 A. Exploiting the capability to measure lines with a very small equivalent width we calculated a new redshift lower limit obtaining $z \geq 0.30$.

$H$ 1722+119: this source $(R \sim 15)$ was studied by Allen et al. (1982) through spectrophotometry carried out with the 4 m class Anglo-Australian telescope in the 80's confirming its bona-fide BL Lac nature (Allen et al. 1982). This object is also a well known strong radio (Healey et al. 2007), X-ray (Donato et al. 2005) and $\gamma$-ray emitter (see e.g. Abdo et al. 2010). The source has been recently discovered during a flare by MAGIC collaboration (Cortina 2013) in the TeV band. Previous spectroscopical study with $8 \mathrm{~m}$ class telescope (Sbarufatti et al. 2006) confirmed that $\mathrm{H} 1722+119$ exhibits a completely featureless spectrum and put this object at $z \geq 0.17$. We infer a tighter lower limit to the redshift of $z>0.35$ from the minimum $E W_{\text {min }}$ measured on 


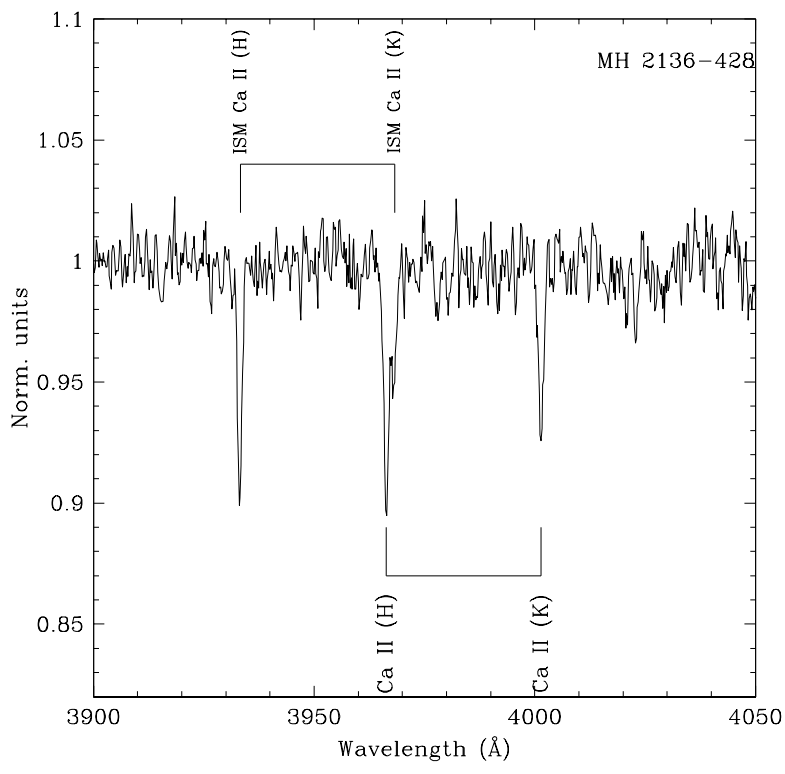

Fig. 3. MH 2136-428 Ca II intervening system arising in the nearby galaxy NGC 7097.

the spectrum. We also remark that the source is unresolved in the image obtained with HST/WFPC3 (Urry et al. 2000).

MH 2136-428: this is a bright BL Lac object $(R \sim 15)$, which was discovered through strong optical variability $(\Delta m \sim 1.0)$ from the optical survey carried with the UK 1.2 Schmidt telescope (Hawkins et al. 1991). Spectroscopic studies (Hawkins et al. 1991; Sbarufatti et al. 2006; and Shaw et al. 2013) failed to detect intrinsic or intervening features. In our spectrum, there are no significant intrinsic emission or absorption lines down to $E W_{\text {min }}=0.06$ that set a lower limit of the redshift $z \geq 0.50$ (see Sect. 2). We note the presence of an intervening absorption system due to Ca II at $\lambda \lambda 3966.404001 .20$ (see Figs. 6-17 and 3), which corresponds to a radial velocity of $2450 \pm 50 \mathrm{~km} \mathrm{~s}^{-1}$. We note that the $\mathrm{H}$ line is blended with the ISM Ca II $\mathrm{K}$ line. The EWs of the intervening absorption (obtained from deblending of the line for the $H$ band) are $E W=0.15 \pm 0.05 \AA$ and $0.10 \pm 0.02 \AA$. We searched for low redshift galaxies in the field of MH 2136-428 associated with the absorption. The most plausible object responsible for this absorption is the halo of the early type NGC $7097\left(v_{r} \sim 2400-2600 \mathrm{~km} \mathrm{~s}^{-1}\right)$ at $\sim 100 \mathrm{Kpc}$ from the target (see Fig. 4). The target was observed by HST/ACS (Proposal ID: 9494, PI: Paolo Padovani) in a program for studying jet features in blazars. The unpublished image (see Fig. 4) shows the presence of a faint $\left(m_{G 1} \sim 21\right)$ spiral galaxy at $\sim 3^{\prime \prime}$ northwest from the BL Lac. The faintness of G1 suggests that it is a background galaxy. We used the HST image to search for emission of host galaxy of the BL Lac. The analysis performed with AIDA (Uslenghi \& Falomo 2011) shows that the target is unresolved, which is consistently with the relatively high redshift deduced from our spectroscopy.

PKS 2254-204: this object $(R \sim 17)$ is a strongly polarised (Impey \& Tapia 1990) featureless BL Lac (Véron-Cetty \& Véron 2001). More recent high quality spectra confirm the featureless emission (see Sbarufatti et al. 2006). Our observation allows us to further search for very faint features (down to $E W \sim 0.10 \AA$ ) over a wider spectral range. In spite of this improvement, the spectrum remains lineless, which indicates a high nuclear-to-host galaxy emission. Based on this $E W_{\text {lim }}$ we set a redshift limit of $z>0.65$.

\section{Discussion and conclusions}

We obtained high-quality spectra from near-UV to near-IR of a small sample of very bright BL Lac objects of unknown redshift. In spite of the significant improvement in terms of the $\mathrm{S} / \mathrm{N}$ and spectral resolution of the new spectra with respect to previous data, we do not detect any intrinsic spectral feature arising from these sources. From the lack of starlight absorptions, we derived stringent lower limits to their redshift, which when combined with the brightness of the objects indicates that these BL Lacs have a very high nuclear to host ratio (see Table 2) compared to the average NHR values $(0.1$ to 1.0$)$ as derived from Urry et al. (2000) and Scarpa et al. (2000). The extreme nucleus absolute magnitude and $\mathrm{N} / \mathrm{H}$ ratio (in particular for $\mathrm{PG} 1553+113$ and $\mathrm{H} 1722+119$ ) may be ascribed to an intrinsic huge luminosity, to a major beaming, or to both effects combined. Assuming the presence of underlying broad emission lines, one can estimate an optical beaming factor $\delta_{\text {opt }}$, which represents the ratio of the non-thermal (jet) to thermal component (disc) of the accreting nucleus. Following Landoni et al. (2013) and supposing a value for $z \sim 0.40$ such that $\mathrm{Mg}$ II $\left(\lambda_{0}=2800\right)$ is in the region of 4000-8000 $\AA$, one can calculate the lower limit of $\delta_{\text {opt }}$ by adopting the minimum observable $E W_{\text {min }}$ (see Table 2). Under this hypothesis, the presence of an extreme beaming is suggested according to values reported in Table 2.

Although the main result of the paper is to fix a lower limit to the $z$ of the objects, one can put also upper limits by exploiting the incidence of $\mathrm{Mg}$ II absorption systems. Considering the distribution of those systems with $E W \geq 0.2 \AA$ (Zhu \& Ménard 2013), we evaluate that the expected number of system per source should to be $\sim 0.8$. In the spectra of our four sources we do not detect absorption systems of $\mathrm{Mg}$ II. Combining this fact with the TeV detection of 2 sources (PG 1553+113 and $\mathrm{H} 1722+119)$ we infer that the upper limit to the redshift should be $z \lesssim 0.50$, in average.

The observations presented in this paper highlight the today capabilities to explore the spectroscopic properties of BL Lac objects and thus the determination of their redshift or tighter lower limits. To make a significant improvement in this field, a major contribution comes from the next generation of extremely large (30-50 m class) telescopes, such as the Thirty Meter Telescope ${ }^{2}$ and European Extremely Large Telescope (E-ELT) ${ }^{3}$. With these new facilities it will become possible to obtain a very high $\mathrm{S} / \mathrm{N}$ spectra of bright BL Lacs and therefore detect the absorption features of their host galaxies under extreme conditions. To exemplify this point we performed simulations of the spectra of our targets as could be secured by an X-Shooter-like instrument $(R \sim 3000)$ coupled to the E-ELT, which includes moderate adaptive optics facility. For the simulation, we assume a plate scale of $0.03^{\prime \prime} \mathrm{px}^{-1}$ and a PSF with $\sim 90 \%$ of encircled energy within an aperture of $3^{\prime \prime}$ to maximise the host galaxy flux inside the aperture. We estimated the $\mathrm{S} / \mathrm{N}$ of each object using the AETC $^{4}$ (Falomo et al. 2011). We produced simulated spectra of the targets, assuming $z=0.50$ and $z=1.00$, in order to evaluate the maximum nucleus to host galaxy flux ratio that allows one to detect the absorption lines of the galaxies (see Fig. 5).

\footnotetext{
2 http://www.tmt.org

3 http://www . eso.org/sci/facilities/eelt/

4 http://aetc.oapd.inaf.it
} 

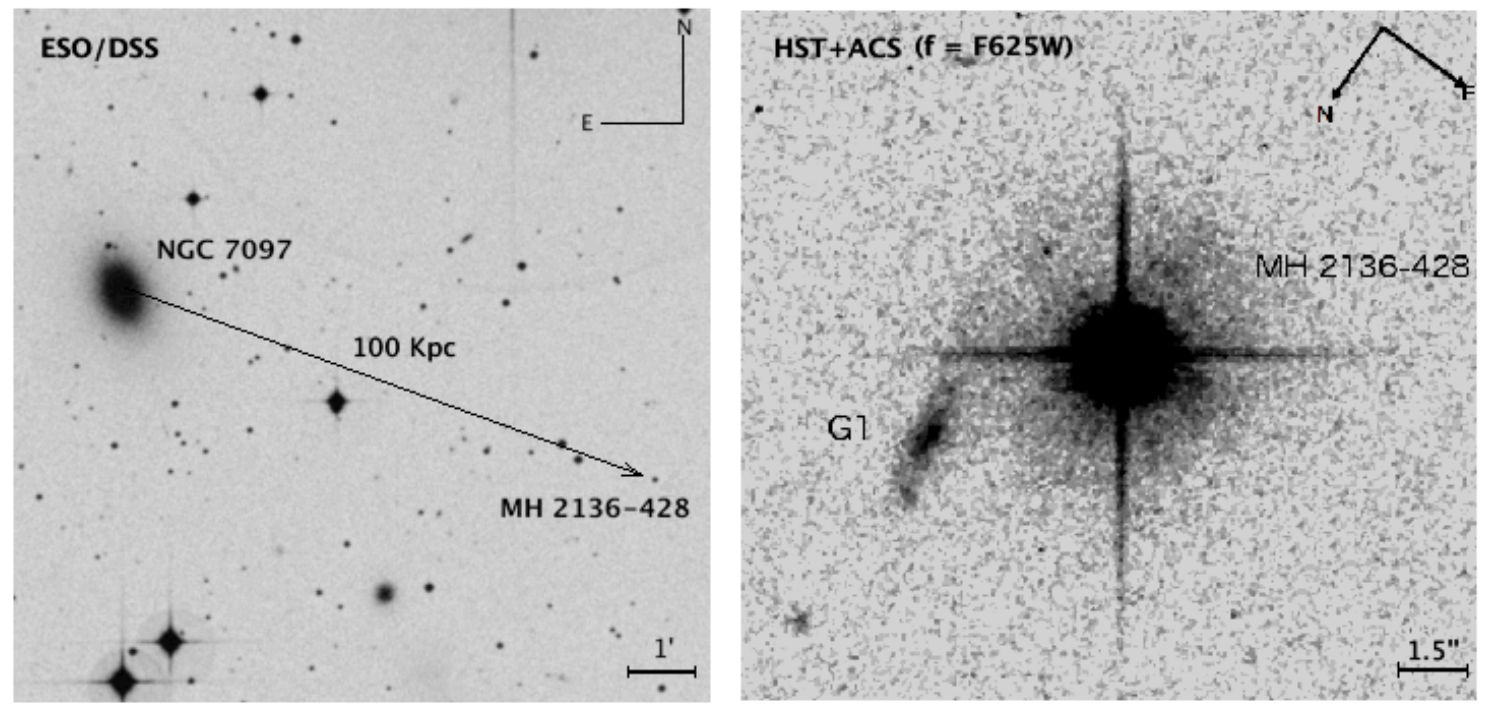

Fig. 4. BL Lac object MH 2136-428. Left panel: ESO/DSS field that shows the galaxy NGC 7097 that is possibly associated to the Ca II absorption system. Right panel: HST+ACS image (integration time is $780 \mathrm{~s} ; f=F 625 W$ ). The image clearly shows the presence of a faint galaxy at $3.2^{\prime \prime} \mathrm{NW}$ from the BL Lac (labelled as G1). The target is unresolved and the faint galaxy (G1) at 3.2" NW is likely a background object.
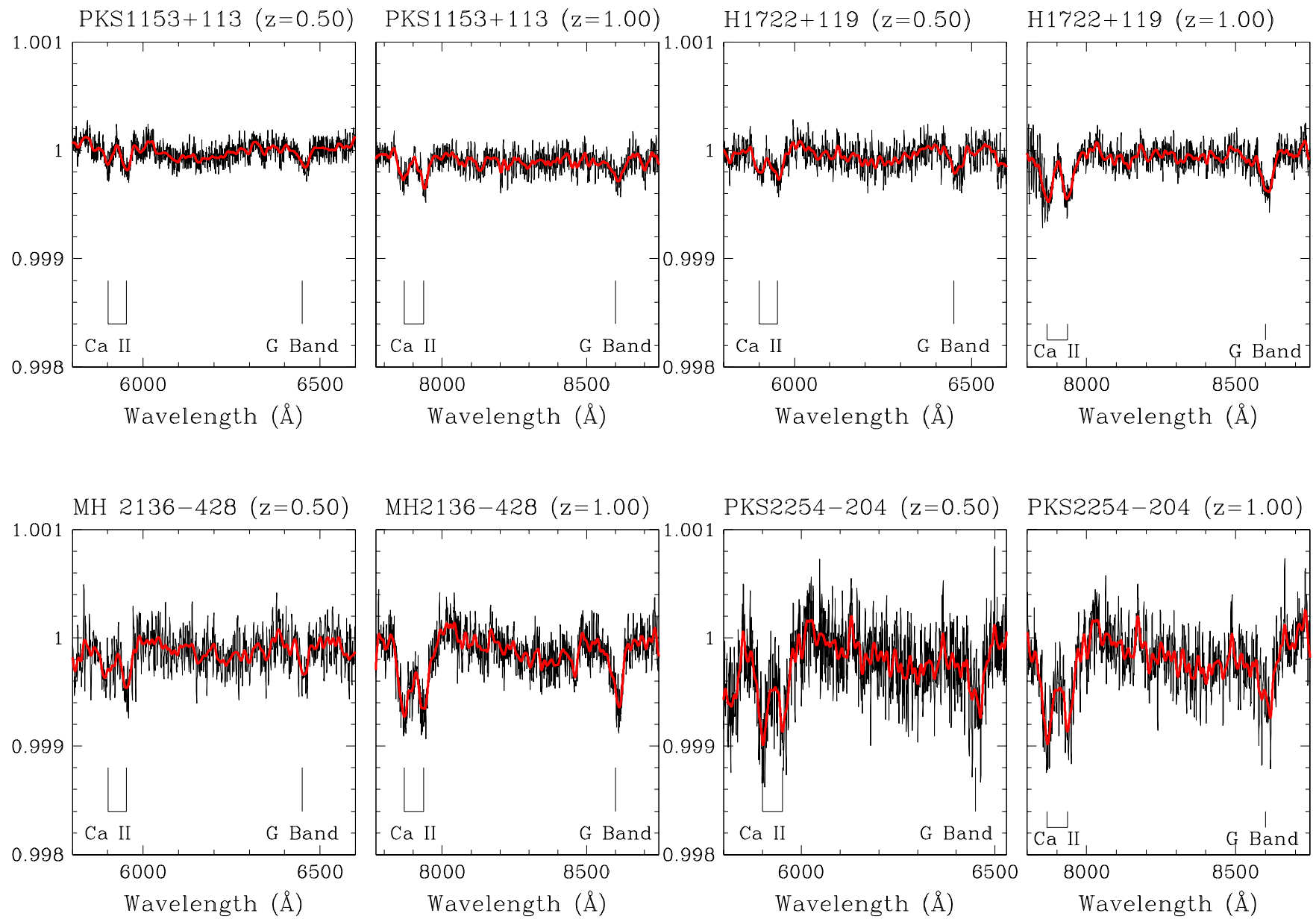

Fig. 5. Simulated spectra of four BL Lacs using E-ELT equipped with an X-Shooter-like instrument ( $t_{\mathrm{exp}}=3600 \mathrm{~s}, R \sim 3000$ ). For each object, we report two cases at $z=0.50$ on the left panel and $z=1.00$ on the right panel. The red solid line is the smoothed spectrum obtained by the adoption of a boxcar filter ( 9 pixels). The spectra represent the maximum N/H flux ratio for which it is possible to detect Ca II and $G$ band absorption lines of the host galaxy (see details in Table 2). 
Table 3. E-ELT X-Shooter-like spectrograph simulation results.

\begin{tabular}{lc|ccccc|ccccc}
\hline \hline Source $^{a}$ & $m_{\text {nuc }}{ }^{b}$ & \multicolumn{3}{|c|}{$z=0.50$} & \multicolumn{4}{c}{$z=1.00$} \\
& & $S N R^{c}$ & Obs. NH $^{d}$ & Int. NH $^{e}$ & $m_{\text {host }}{ }^{f}$ & $M_{R}{ }^{g}$ & $S N R$ & Obs. NH & Int. NH & $m_{\text {host }}$ & $M_{R}$ \\
\hline PG 1553+113 & 13.80 & 5300 & 2000 & 1000 & 21.30 & -21.40 & 6500 & 2500 & 1700 & 21.80 & -23.30 \\
H 1722+119 & 14.90 & 3200 & 700 & 350 & 21.25 & -21.30 & 4000 & 1200 & 800 & 22.20 & -22.70 \\
MH 2136-428 & 15.80 & 2000 & 500 & 250 & 21.80 & -20.80 & 2500 & 800 & 540 & 22.90 & -22.10 \\
PKS 2254-204 & 17.10 & 1000 & 300 & 150 & $22-60$ & -20.10 & 1300 & 600 & 400 & 23.60 & -21.40 \\
\hline
\end{tabular}

Notes. ${ }^{(a)}$ Source simulated with E-ELT X-Shooter-like spectrograph. ${ }^{(b)}$ Apparent nucleus magnitude in $R$ band. ${ }^{(c)}$ Obtained signal-to-noise ratio for $3600 \mathrm{~s}$ of exposure. ${ }^{(d)}$ Observed nucleus-to-host ratio at Ca II H \& K band host galaxy features. ${ }^{(e)}$ Integral nucleus-to-host ratio (assuming a slit loss for an aperture of $3^{\prime \prime}$ diameter). ${ }^{(f)}$ Apparent host magnitude in $R$ band. ${ }^{(g)}$ Absolute $R$ band host magnitude.

Result of the simulations are summarised in Table 3. With these future facilities, it turns out that it will be possible to derive the redshift of these objects under extreme conditions of observed $\mathrm{N} / \mathrm{H}$ ratios from $\sim 300$ to $\sim 2500$ depending on redshift and target brightness. This corresponds to total $\mathrm{N} / \mathrm{H}$ ratios in the range of 100-1700 that are 1-2 order of magnitude higher than the average found for resolved BL Lacs (Urry et al. 2000).

\section{References}

Abdo, A. A., Ackermann, M., Ajello, M., et al. 2010, ApJS, 188, 405 Aleksić, J., Alvarez, E. A., Antonelli, L. A., et al. 2012, ApJ, 748, 46 Allen, D. A., Ward, M. J., \& Hyland, A. R. 1982, MNRAS, 199, 969 Cortina, J. 2013, ATel, 5080, 1

Costamante, L. 2013, Int. J. Mod. Phys. D, 22, 30025

Costamante, L., \& Ghisellini, G. 2002, A\&A, 384, 56

Danforth, C. W., Keeney, B. A., Stocke, J. T., Shull, J. M., \& Yao, Y. 2010, ApJ, 720,976

Donato, D., Sambruna, R. M., \& Gliozzi, M. 2005, A\&A, 433, 1163

Falomo, R., \& Treves, A. 1990, PASP, 102, 1120

Falomo, R., Bersanelli, M., Bouchet, P., \& Tanzi, E. G. 1993, AJ, 106, 11

Falomo, R., Fantinel, D., \& Uslenghi, M. 2011, in SPIE Conf. Ser., 8135
Furniss, A., Williams, D. A., Danforth, C., et al. 2013, ApJ, 768, L31

Goldoni, P., Royer, F., François, P., et al. 2006, in SPIE Conf. Ser., 6269

Green, R. F., Schmidt, M., \& Liebert, J. 1986, ApJS, 61, 305

Hawkins, M. R. S., Veron, P., Hunstead, R. W., \& Burgess, A. M. 1991, A\&A, 248,421

Healey, S. E., Romani, R. W., Taylor, G. B., et al. 2007, ApJS, 171, 61

Impey, C. D., \& Tapia, S. 1990, ApJ, 354, 124

Landoni, M., Falomo, R., Treves, A., et al. 2012, A\&A, 543, A116

Landoni, M., Falomo, R., Treves, A., et al. 2013, AJ, 145, 114

Prandini, E., Bonnoli, G., Maraschi, L., Mariotti, M., \& Tavecchio, F. 2010, MNRAS, 405, L76

Rau, A., Schady, P., Greiner, J., et al. 2012, A\&A, 538, A26

Sbarufatti, B., Treves, A., \& Falomo, R. 2005a, ApJ, 635, 173

Sbarufatti, B., Treves, A., Falomo, R., et al. 2005b, AJ, 129, 559

Sbarufatti, B., Treves, A., Falomo, R., et al. 2006, AJ, 132, 1

Scarpa, R., Urry, C. M., Falomo, R., Pesce, J. E., \& Treves, A. 2000, ApJ, 532, 740

Shaw, M. S., Romani, R. W., Cotter, G., et al. 2013, ApJ, 764, 135

Treves, A., Falomo, R., \& Uslenghi, M. 2007, A\&A, 473, L17

Urry, C. M., Scarpa, R., O’Dowd, M., et al. 2000, ApJ, 532, 816

Uslenghi, M., \& Falomo, R. 2011, in SPIE Conf. Ser., 8135

Vernet, J., Dekker, H., D'Odorico, S., et al. 2011, A\&A, 536, A105

Véron-Cetty, M.-P., \& Véron, P. 2001, A\&A, 374, 92

Zhu, G., \& Ménard, B. 2013, ApJ, 770, 130

Pages 8 to 19 are available in the electronic edition of the journal at http://www . aanda.org 

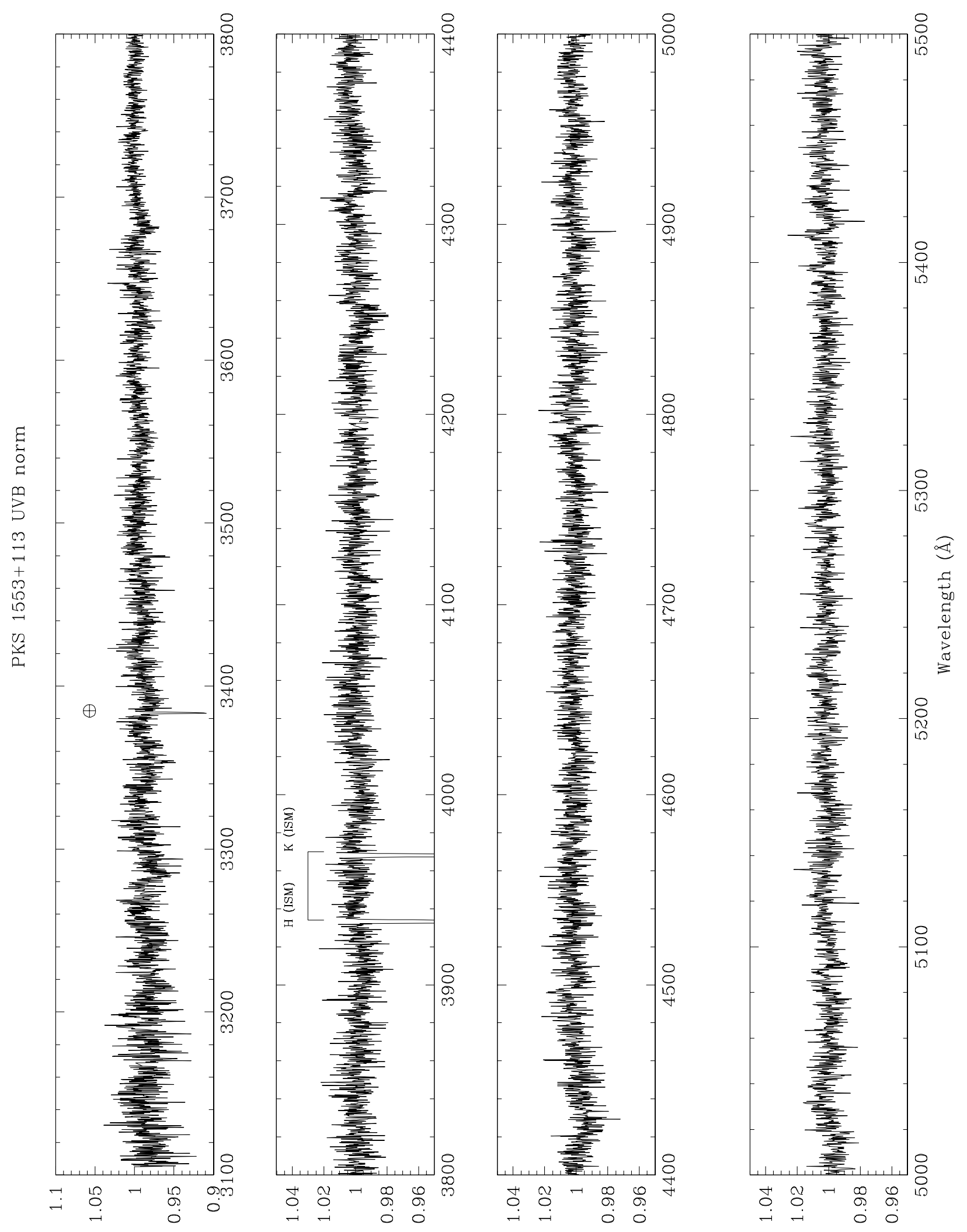

Fig. 6. PG 1553+113 X-Shooter normalized UVB spectrum. 

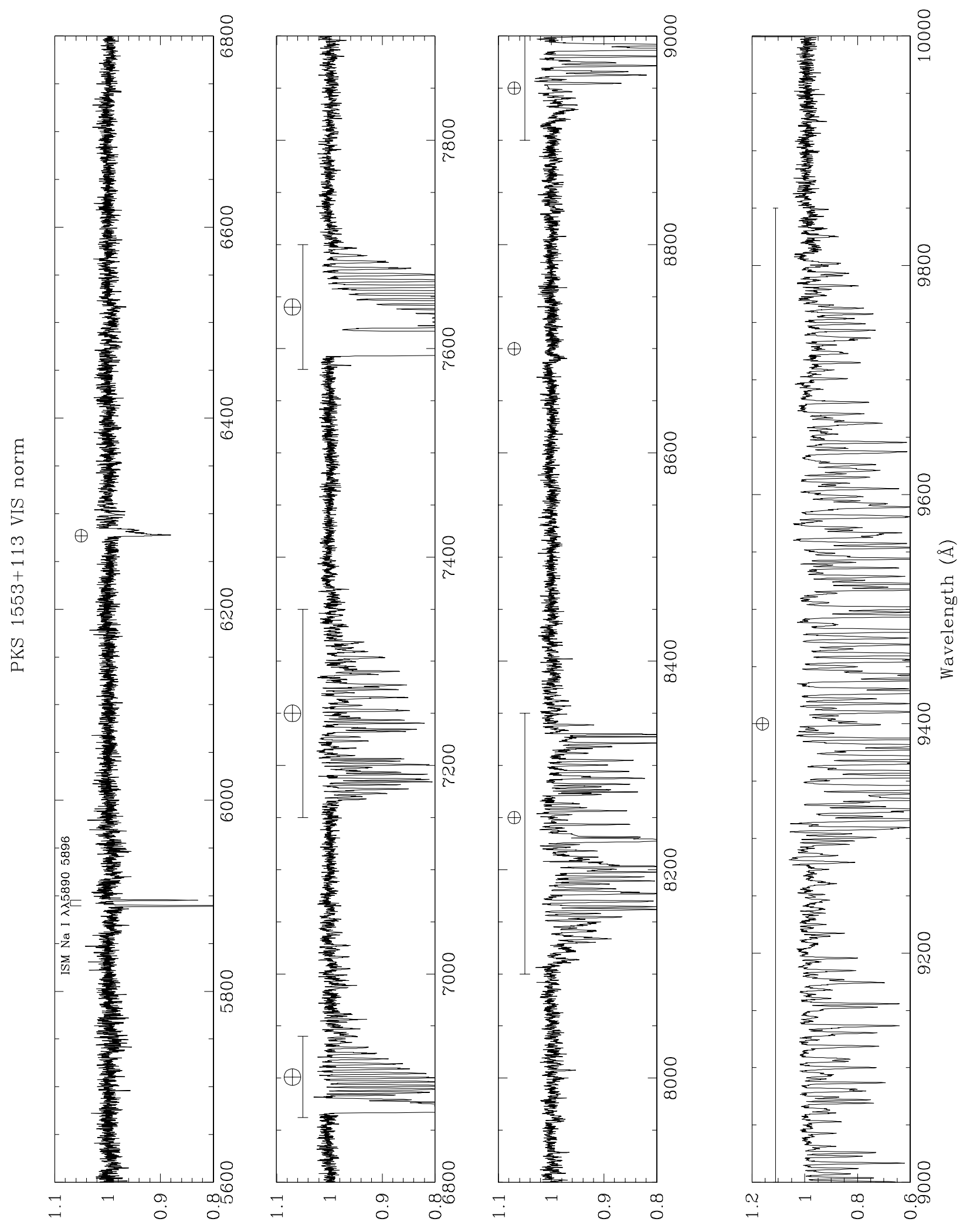

Fig. 7. PG 1553+113 X-Shooter normalized optical spectrum. 

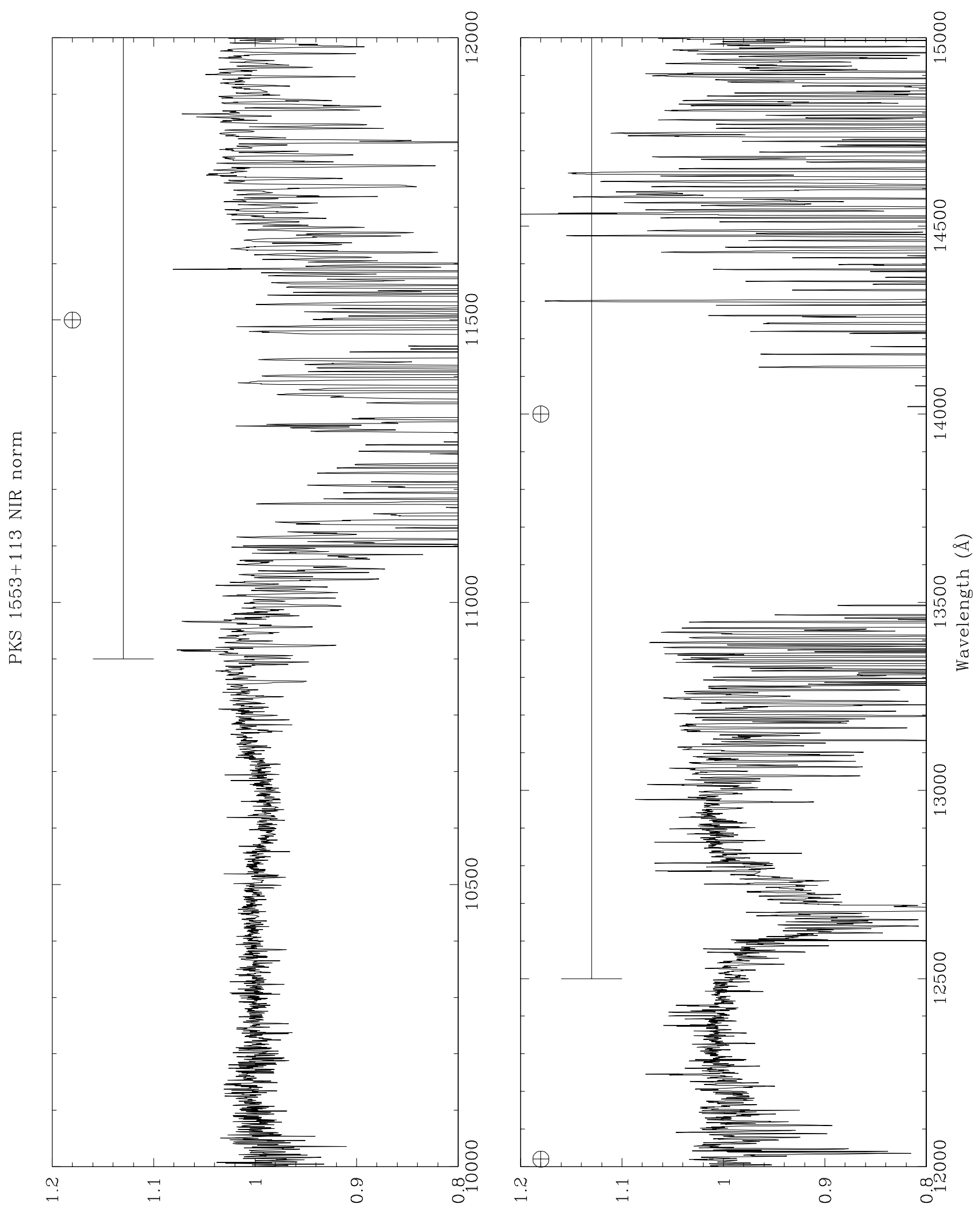

Fig. 8. PG 1553+113 X-Shooter normalized near-IR spectrum. 

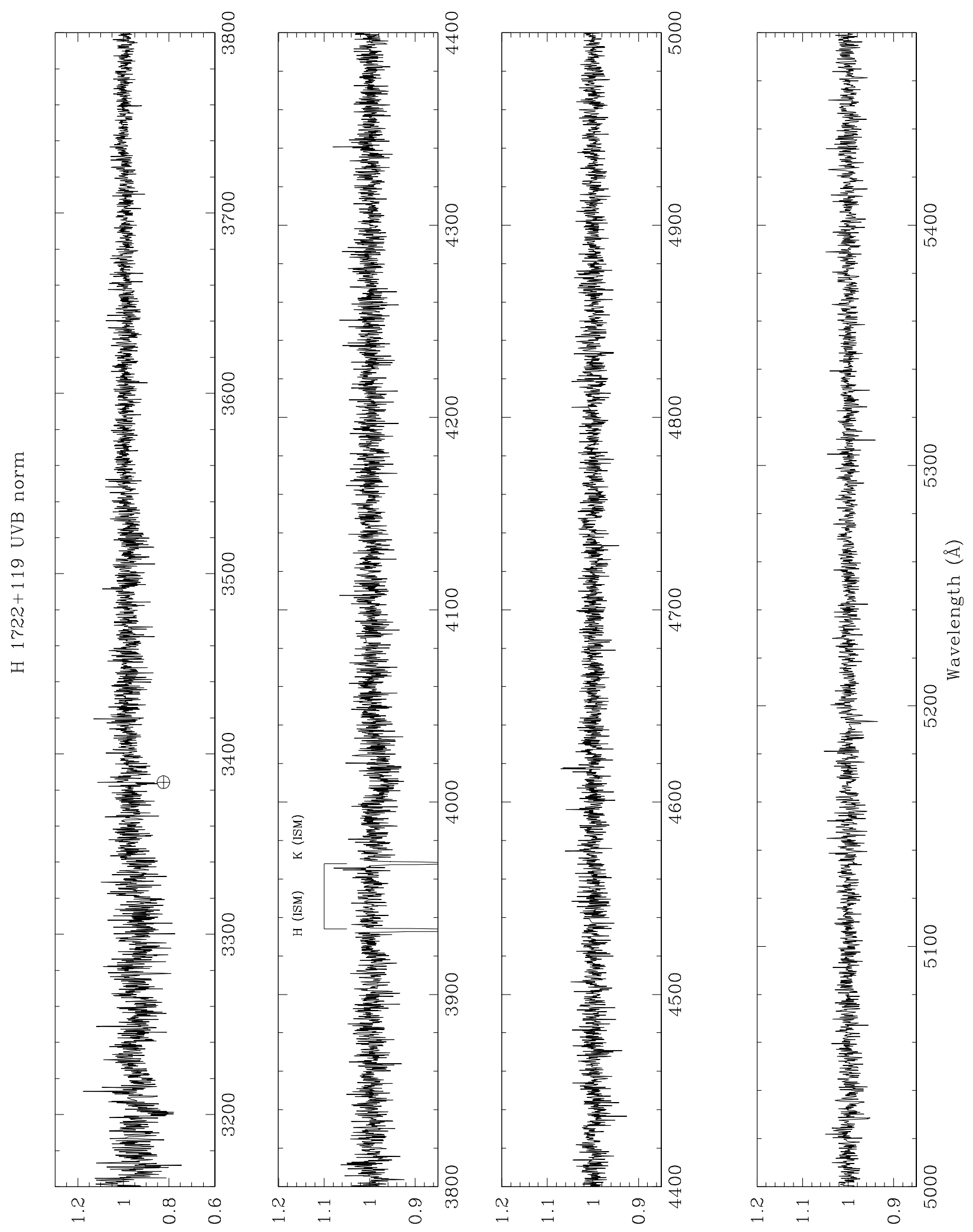

Fig. 9. H 1722+119 X-Shooter normalized UVB spectrum. 

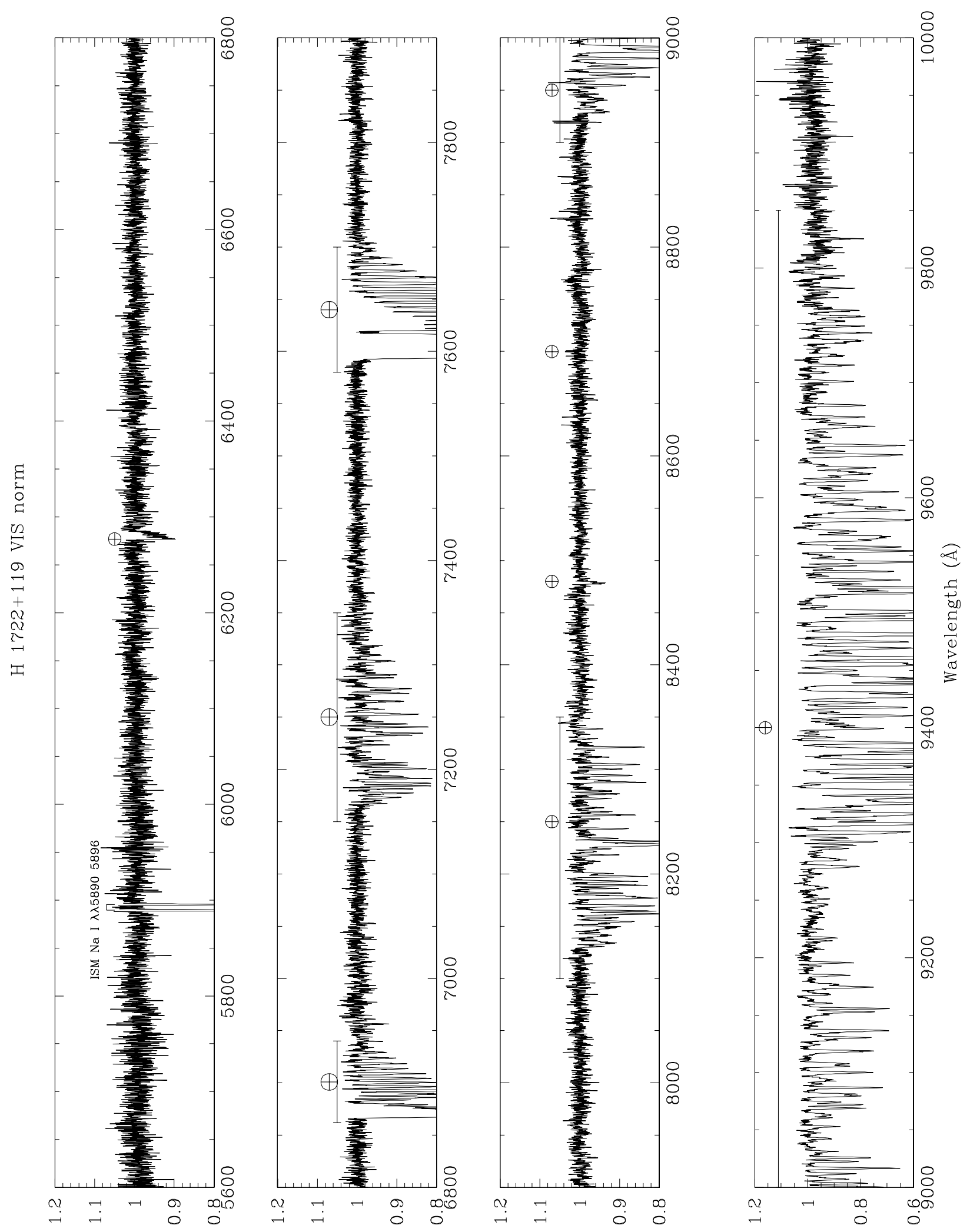

Fig. 10. H 1722+119 X-Shooter normalized optical spectrum. 

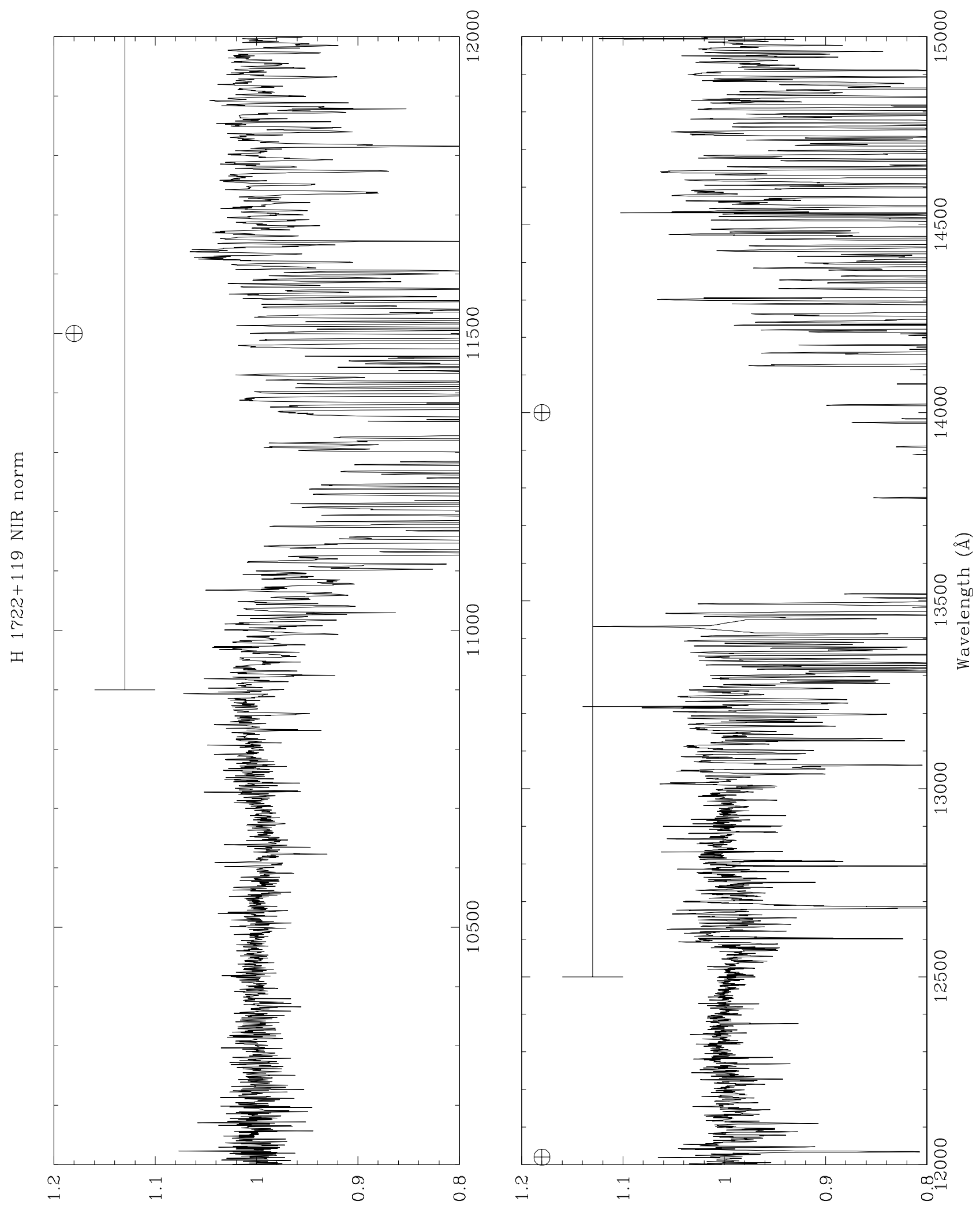

Fig. 11. H 1722+119 X-Shooter normalized near-IR spectrum. 


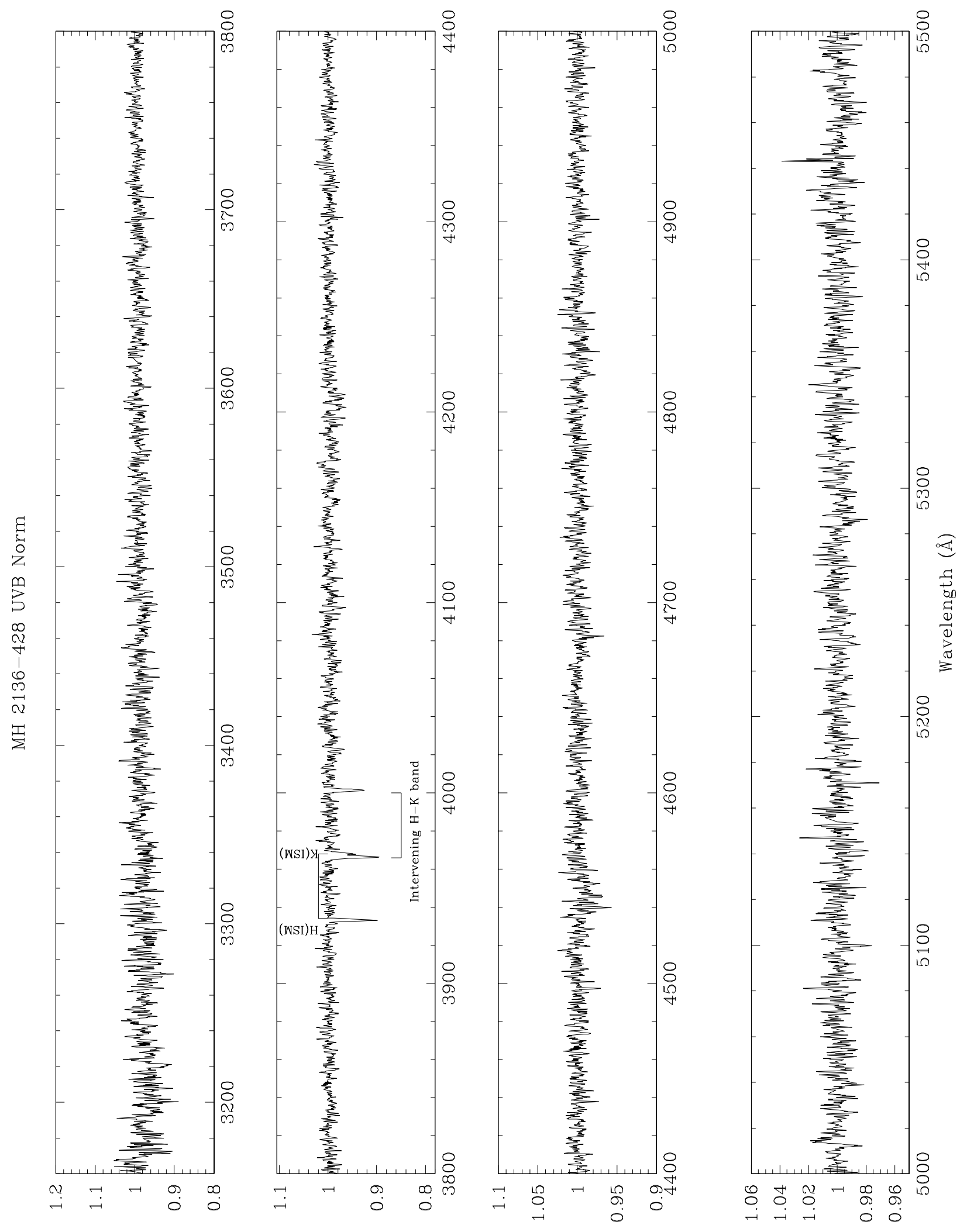

Fig. 12. MH 2136-428 X-Shooter normalized UVB spectrum. 

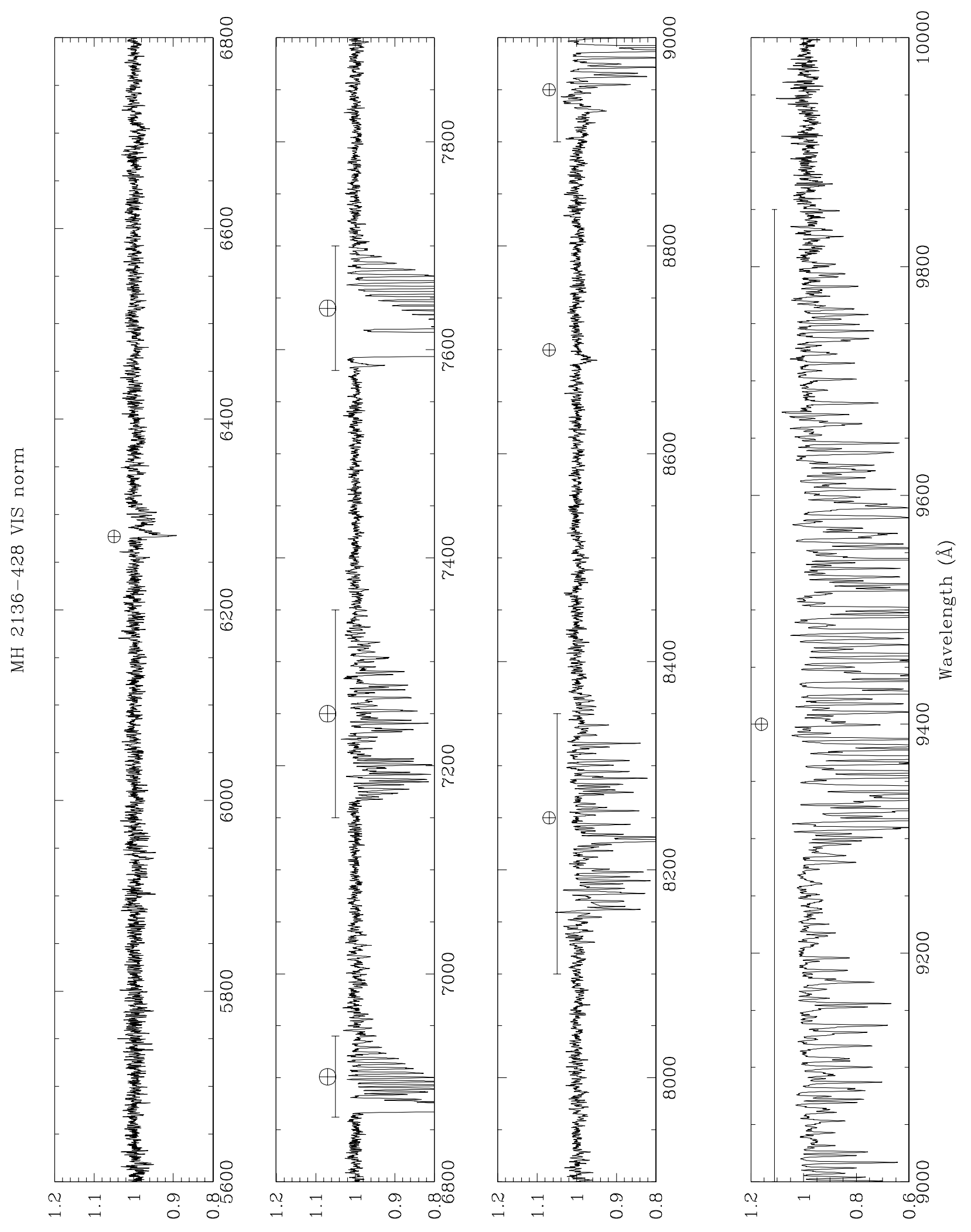

Fig. 13. MH 2136-428 X-Shooter normalized optical spectrum. 

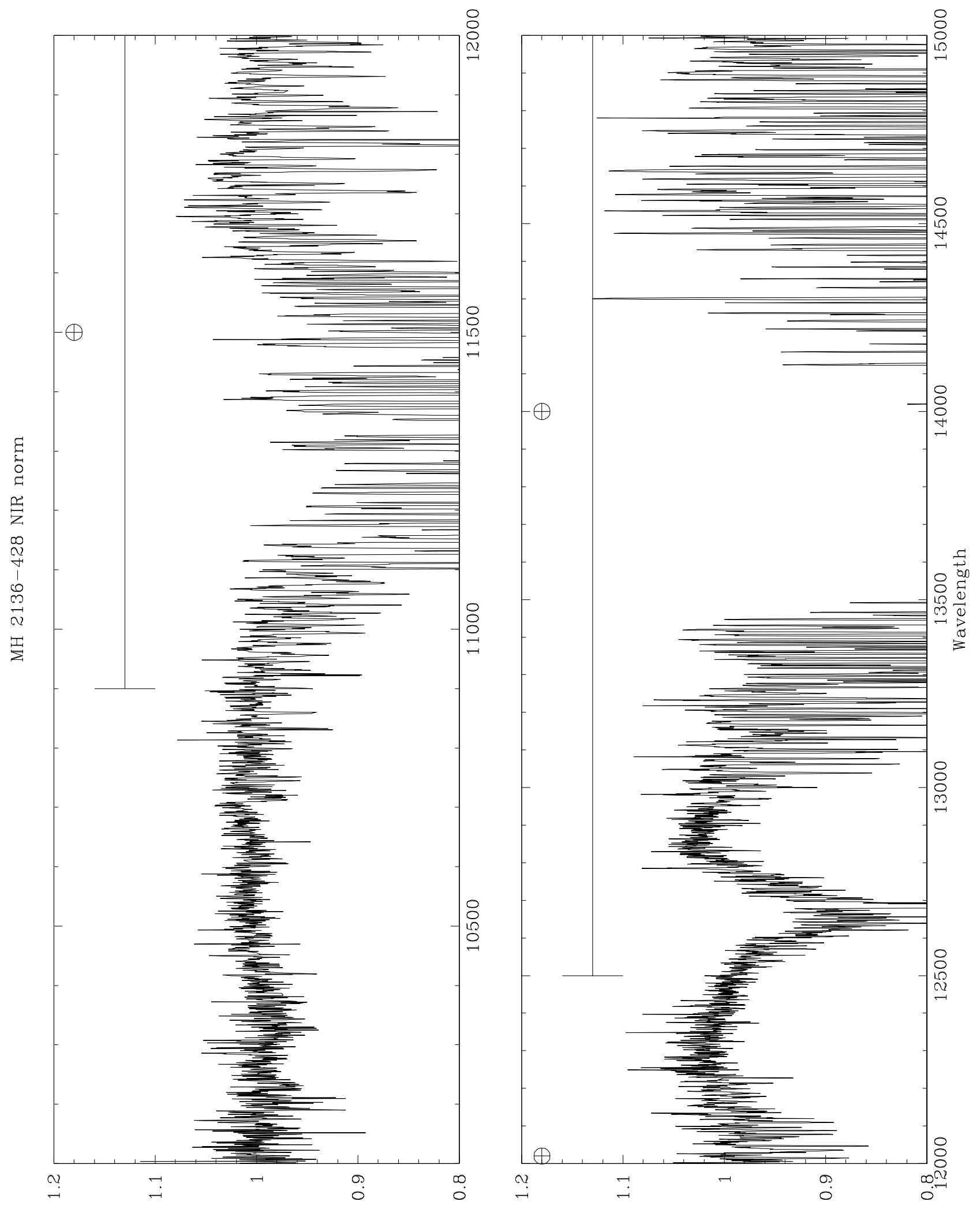

Fig. 14. MH 2136-428 X-Shooter normalized near-IR spectrum. 

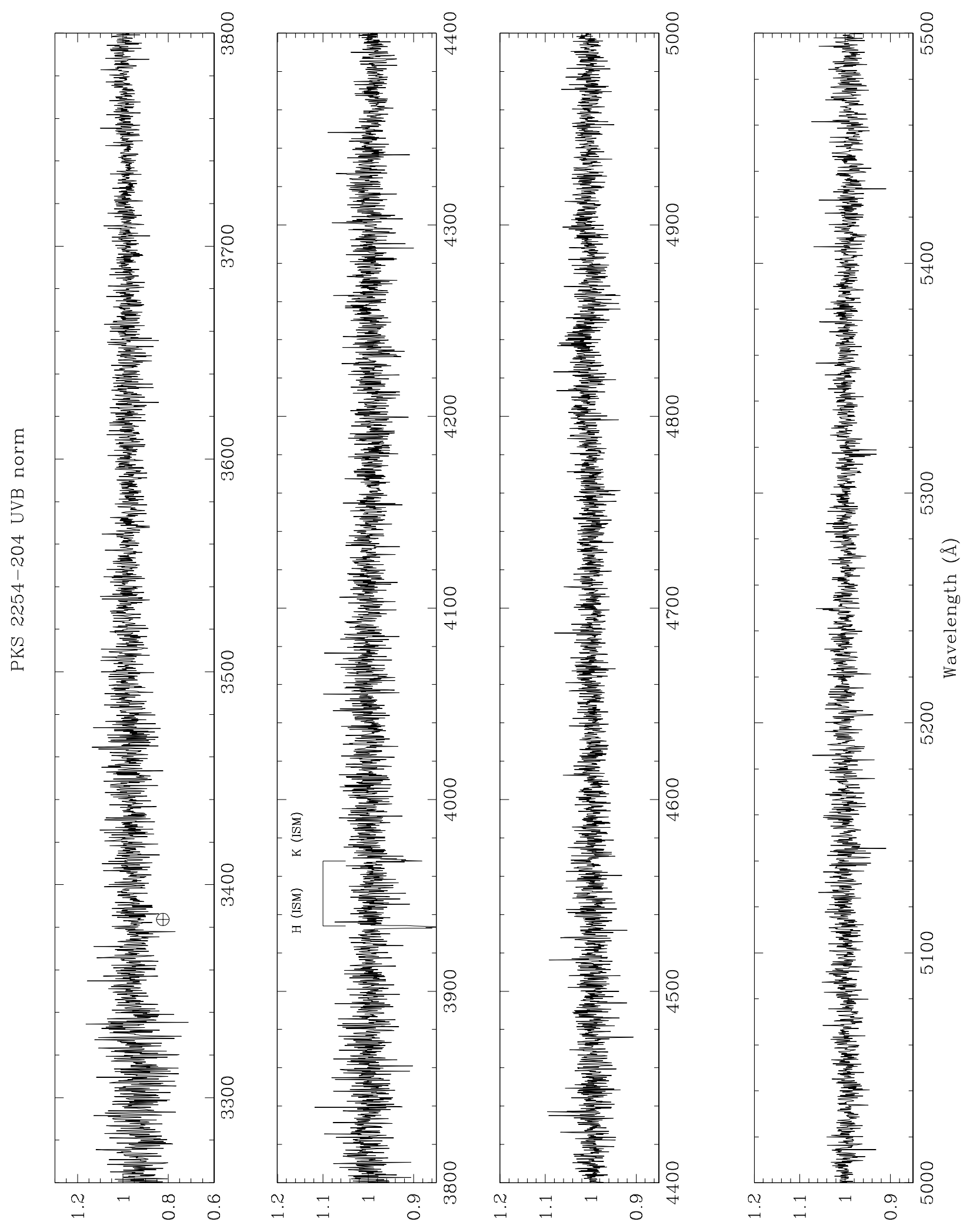

Fig. 15. PKS 2254-204 X-Shooter normalized UVB spectrum. 

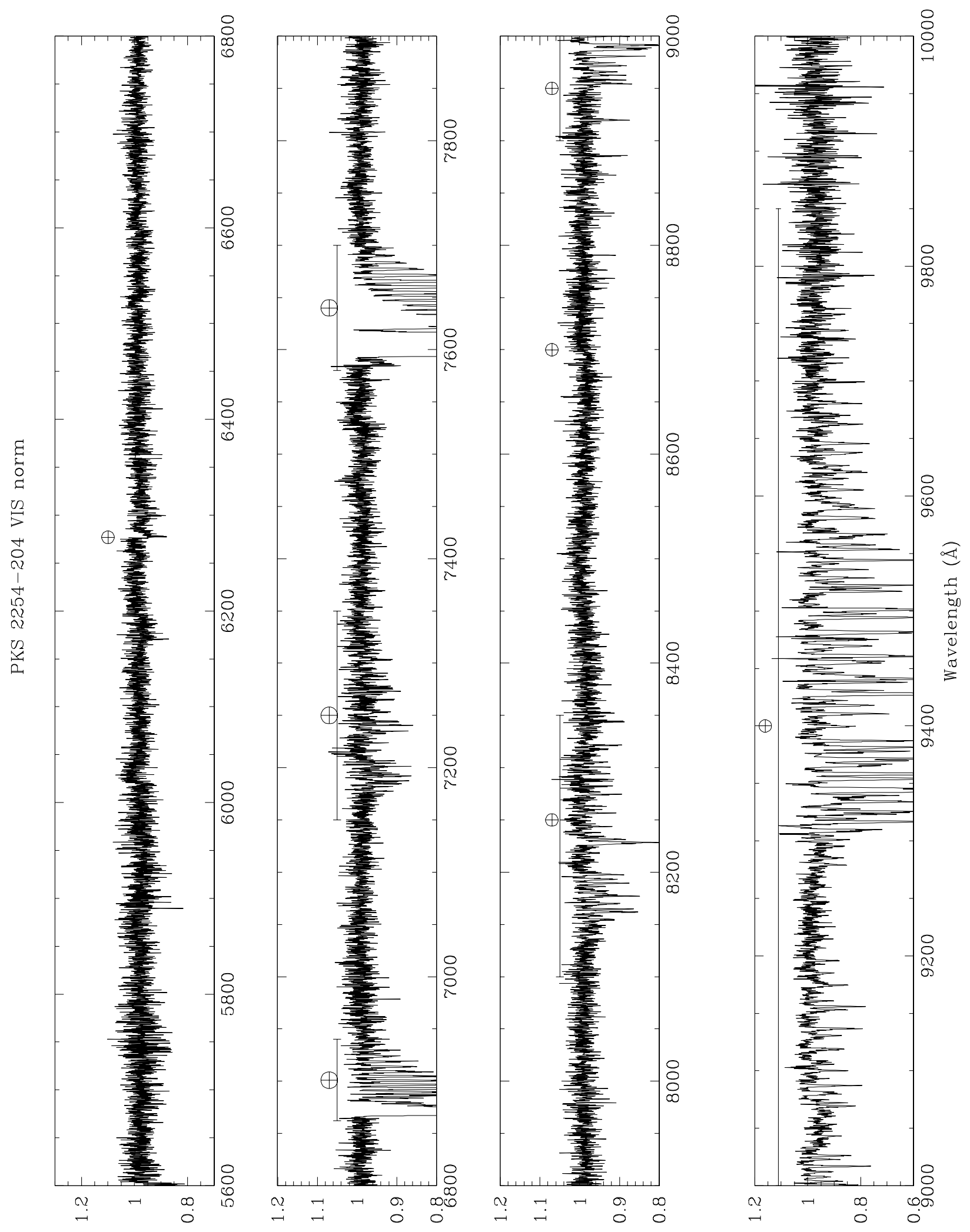

Fig. 16. PKS 2254-204 X-Shooter normalized optical spectrum. 

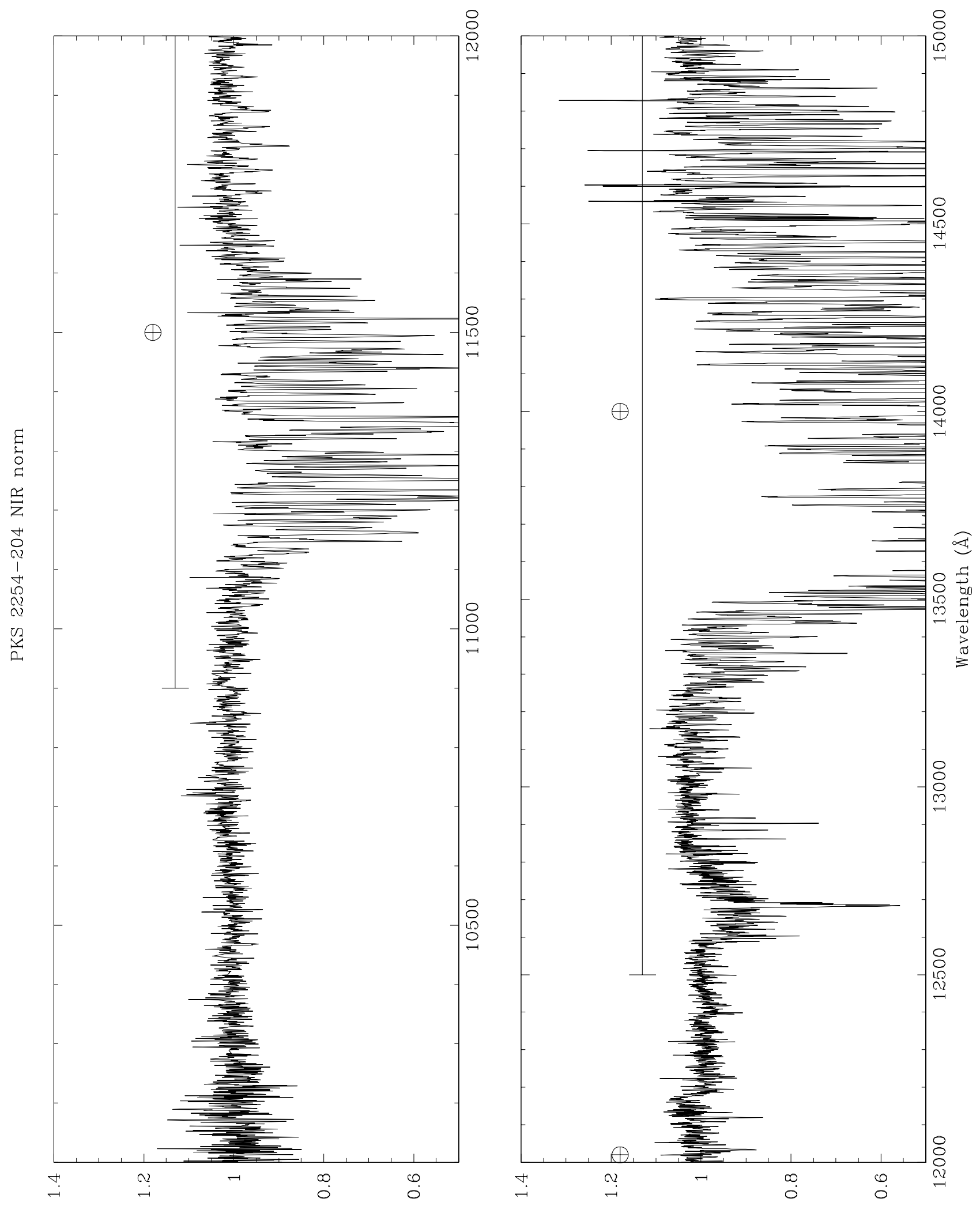

Fig. 17. PKS 2254-204 X-Shooter normalized near-IR spectrum. 